\title{
Correlation between polychromatic image quality metrics to predict visual acuity in pseudophakic patients
}

\author{
Jesús Armengol,,${ }^{1, *}$ Nuria Garzón, ${ }^{2}$ Fidel Vega, ${ }^{1}$ Irene Altemir, ${ }^{3}$ and María S. Millán. ${ }^{1}$ \\ ${ }^{1}$ Departament d’Òptica i Optometria. Universitat Politècnica de Catalunya. Barcelona Tech. 08222. Terrassa. Spain. \\ ${ }^{2}$ Miranza IOA. 28003 Madrid, Spain and Optometry and Vision Department. UCM University. 28037 Madrid, Spain. \\ ${ }^{3}$ Miguel Servet University Hospital. Ophthalmology Department. 50009 Zaragoza, Spain.
}

\begin{abstract}
The optical quality of three multifocal intraocular lenses (MIOLs) was measured in vitro using the area under the modulation transfer function (MTFa) and energy efficiency (EE). We evaluated those metrics through-focus (TF) by axially scanning the image space with three wavelengths (R, G, B). High linear correlation between MTFa and EE was found for all cases. Correlations between optical in vitro results (polychromatic TF-MTFa and TF-EE) and clinical visual acuity (VA) defocus curves for the three MIOLs allowed us to derive mathematical expressions. Both metrics proved to be equivalent to predict postoperative VA.
\end{abstract}

\section{Introduction}

Cataract surgery is evolving from a solution to visual impairment to a refractive solution. The use of multifocal intraocular lenses (MIOLs) is increasingly widespread to improve the refractive performance.

By analyzing the through focus optical quality of MIOLs, Marsack et al. [1] concluded that MTFa and EE -through the light-in-the-bucket (LIB) estimate- were among the six PSF-based metrics that accounted for over $70 \%$ of the variance in postoperative LogMar VA.

\section{Method}

To obtain in vitro the images produced by the MIOLs, we used the on-bench eye model described in detail in [2]. The system met the standards of the International Organization for Standardization, ISO 11979-2: 2014. We have studied three MIOLs: a FineVision PODF (Physiol), a Versario 3F (Bausch+Lomb), and a Tecnis Symfony (J\&J Vision), all of them with a diffractivebased design and 20D base power. These lenses have an addition between $+3 \mathrm{D}$ and $+3.5 \mathrm{D}$ for the near focus and between $+1.5 \mathrm{D}$ and $+1.75 \mathrm{D}$ for the intermediate focus. We measured MTFa and EE to study the trough-focus MIOL performance. MTFa is the area under the MTF curve for the range of frequencies of interest in vision. We measured the MTF in two perpendicular directions by placing a cross-line object of 10 microns slit width. We integrated the average MTF up to the $50 \mathrm{cyc} / \mathrm{mm}$ $(\approx 15$ cyc/degree). We imaged a pinhole and measured EE from the LIB estimation as reported in detail elsewhere [3]. Each MIOL image space was TF scanned sequentially with blue (B) $455 \mathrm{~nm}$, green (G) $530 \mathrm{~nm}$ and red (R) $650 \mathrm{~nm}$ wavelengths.
The clinical data were obtained from 102 eyes of 52 patients recruited in two clinical trials carried out at two ophthalmology centers. Both studies were prospective, consecutive and non-randomized and followed the tenets of the declaration of Helsinki. The patients underwent bilateral and symmetrical cataract surgery followed by MIOL implantation into the capsular bag. Previously, they had been fully informed about the study and signed a consent form. The local ethics committee approved the corresponding trial. Monocular defocus VA curves from $-5.00 \mathrm{D}$ to $+3.00 \mathrm{D}$, with the patients wearing their best distance correction, were measured during the last postoperative follow up.

\section{Results}

Figure 1 shows the TF-MTFa (A) and TF-EE (B) curves for a MIOL (FineVision) with $3 \mathrm{~mm}$ pupil. Both groups of curves appear to be very similar for each wavelength. The polychromatic TF curves (black line) are computed from linear combination of the RGB curves weighted by their photometric coefficients.

Figure 2 depicts, for every MIOL and wavelength, the (MTFa, EE)x pairs at each defocus position $\mathrm{x}(\mathrm{D})$. The figure reveals very good linear correlation between MTFa and EE $\left(\mathrm{R}^{2}>0.96\right)$.

Following the methodology described in closely related studies [4,5], Figure 3 shows in blue dots the clinical $\mathrm{VA}(\mathrm{x})$ outcomes versus a polychromatic (poly) function for every defocus or image vergence (x): (A) MTFa_poly(x) and (B) EE_poly(x). We fit the points of Figures $3(\mathrm{~A}, \mathrm{~B})$ to either exponential functions (green solid line) (Eqs. 1, 2).

${ }^{*}$ Corresponding author: jesus.armengol@upc.edu 


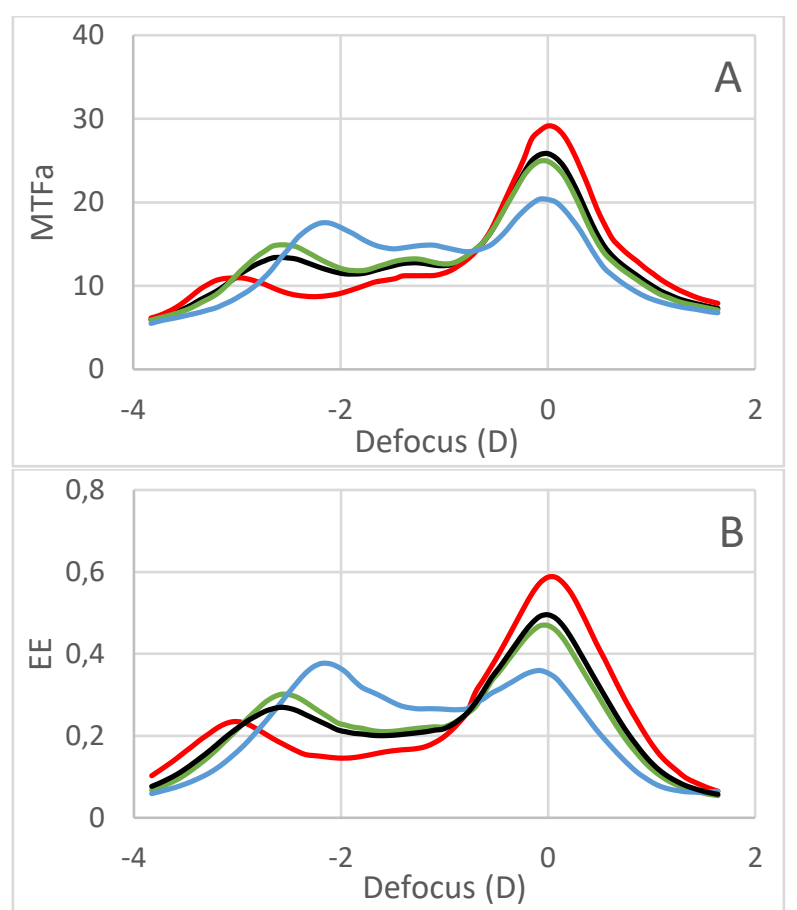

Fig. 1. Monochromatic R, G and B MTFa (A) and EE (B) versus defocus (at spectacle plane) obtained with $3 \mathrm{~mm}$ pupil for the FineVision MIOL. Polychromatic functions in black.

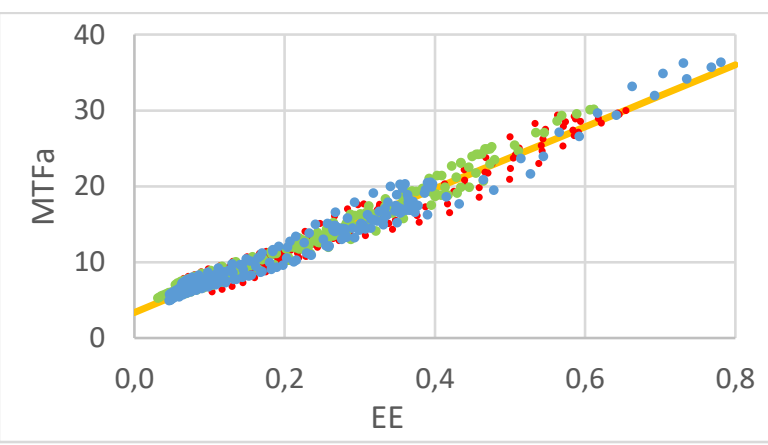

Fig. 1. MTFa vs. EE for the three MIOLs with $3 \mathrm{~mm}$ pupil and three wavelengths (R, G, B dots). Best linear fit (yellow).

The mathematical expressions and their correlation coefficients $\left(\mathrm{R}^{2}\right)$ are

$$
\begin{array}{ll}
V A=1.828 * e^{-0.230^{*} M T F a_{\text {poly }}}+0.014 & R^{2}=0.94 \\
V A=0.707 * e^{-7.746^{*} E E_{\text {poly }}} & R^{2}=0.90
\end{array}
$$

\section{Discussion}

MTFa and EE are objective metrics to assess in-vitro the optical image quality of MIOLs; the larger the value of any of these two metrics, the better the optical quality.

Our experimental results (Fig. 2) show a clear linear correlation between MTFa and EE values $\left(\mathrm{R}^{2}>0.96\right)$. Prior research [4-7] showed the potential of MTFa as a preclinical metric for predicting average postoperative VA at different defocus values. In this work we have proved the ability of the EE metric to predict clinical VA outcomes of patients implanted with three different designs of MIOLs.

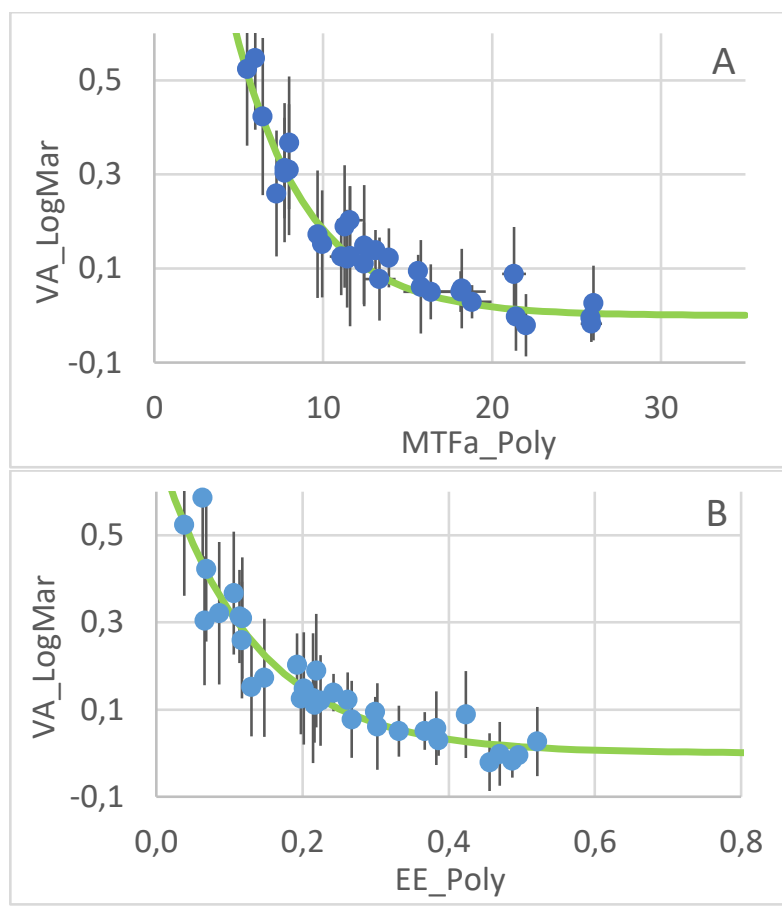

Fig. 3. Clinical Visual Acuity versus polychromatic (A) MTFa_Poly and (B) EE_Poly for $3 \mathrm{~mm}$ pupil (blue dots). Exponential fits (green line).

The results show, in agreement with former works $[4,5,7]$, non-linear relationships between VA and both, MTFa_poly and EE_poly. The mathematical functions that fit the data of Figure 3 with high correlation coefficients, $\mathrm{R}^{2}=0.94$ for VA vs MTFa_poly (Eq. 1) and $\mathrm{R}^{2}=0.90$ for VA vs EE_poly (Eq. 2), clear a path to predict average VA of pseudophakic patients from objective on-bench quality metrics. In the conditions of our study, TF-EE is a very useful optical metric that can replace $\mathrm{MTFa}$ as a preclinical quality parameter.

\section{References}

1. J. D. Marsack, L. N. Thibos, and R. A. Applegate. J. Vis. 4(4), 322-328 (2004).

2. F. Vega, F. Alba-Bueno, and M. S. Millan. J. Eur. Opt. Soc. Rapid Publ. 9, 14002 (2014).

3. M. S. Millán, F. Vega, and I. Ríos-López. Investig. Ophthalmol. Vis. Sci. 57(4), 2021-2028 (2016).

4. A. Alarcon, C. Canovas, R. Rosen, H. Weeber, L. Tsai, K. Hileman, and P. Piers. Biomed. Opt. Express 7(5), 1877 (2016).

5. F. Vega, M. S. Millán, N. Garzón, I. Altemir, F. Poyales, and J. M. Larrosa. Biomed. Opt. Express 9(10), 4893 (2018).

6. J. Fernández, M. Rodríguez-Vallejo, J. Martínez, N. Burguera, and D. P. Piñero. J. Refract. Surg. 35(12), 789795 (2019).

7. G. Cardona, F. Vega, M. A. Gil, C. Varón, J. A. Buil, and M. S. Millán. Eur. J. Ophthalmol. 28(1), 36-41 (2018).

\section{Funding}

Spanish Ministerio de Economía y Competitividad y fondos FEDER (DPI2016-76019-R). 